INVESTIGACIÓN

\title{
CARACTERIZACIÓN DE LA TIPOLOGÍA DE FALLOS PRESENTADOS EN LOS PAVIMENTOS DE LA CIUDAD DE OCAÑA NORTE DE SANTANDER
}

\section{CHARACTERIZATION OF TYPES OF FAILURES ON PAVEMENTS OF OCAÑA CITY - NORTH SANTANDER}

\author{
Msc. Romel Jesús Gallardo Amaya ${ }^{a}$, Ing. Leidy Johana Quintero Lemus ${ }^{\mathrm{b}}$, Ing. Oscar Andres \\ Cuanalo Campo ${ }^{\mathrm{c}}$ \\ ${ }^{a}$ Universidad Francisco de Paula Santander Ocaña, Grupo de Investigación en Geotecnia y \\ Medio Ambiente GIGMA. Vía Acolsure, Sede el Algodonal, Ocaña, Norte de Santander, \\ Colombia, rigallardoa@ufpso.edu.co \\ ${ }^{\mathrm{b}}$ Universidad Francisco de Paula Santander Ocaña, Grupo de Investigación en Geotecnia y \\ Medio Ambiente GIGMA. Vía Acolsure, Sede el Algodonal, Ocaña, Norte de Santander, \\ Colombia, ljquinterole@ufpso.edu.co
${ }^{\mathrm{c}}$ Universidad Francisco de Paula Santander Ocaña, Grupo de Investigación en Geotecnia y Medio Ambiente, GIGMA Rafael Alduci 228, Puebla, México, oscarcuanalo@ hotmail.com

Fecha de recepción: 01-03-2017

Fecha de aprobación: 15-06-2017

\begin{abstract}
Resumen: Los fallos en los pavimentos se presentan por el deterioro progresivo que les ocurre con el tiempo. Una forma de identificar los fallos es mediante una inspección visual, es así como en esta investigación se inspeccionaron varios tramos de las principales vías urbanas del municipio de Ocaña, Norte de Santander. La información se consolidó mediante formatos en donde se registraron coordenadas, anchos del carril, nivel de severidad, observaciones y registro fotográfico de los fallos. Una vez tabulada la información se encontró que fueron analizados $9.277 \mathrm{ml}$ de tramos viales, de los cuales $3.051 \mathrm{ml}$ (32.89\%) tienen pavimento flexible y $6.226 \mathrm{ml}$ $(67.11 \%)$ pavimento rígido. En los flexibles las tipologías de fallos más representativas fueron piel de cocodrilo y baches y, para el caso de los rígidos fueron las grietas y desintegración. El grado de severidad de los fallos mencionados, tanto para el caso de los pavimentos flexibles como rígidos es de medio a alto; siendo esto un indicador de los niveles de deterioro que poseen los pavimentos en los sectores viales analizados. Muchos de los fallos pueden estar asociados a deficiencias en la estructura de los pavimentos y condiciones inadecuadas de los suelos de subrasante.
\end{abstract}

Palabras clave: Fallo, inspección visual, Pavimento, severidad, deterioro. 


\begin{abstract}
Pavement failures are due to the progressive deterioration with time. A way to identify theses failures is through a visual inspection; that is how in this research several sectors of the main urban roads of Ocaña city were inspected. The data were consolidated using forms where coordinates, lane width, severity level, observations and photographic failure registry were annotated. Once the data were tabulated, it was found that $9227 \mathrm{~m}$ of roads were analyzed, among which $3051 \mathrm{~m}(32.89 \%)$ have flexible pavement and $6226 \mathrm{~m}(67.11 \%)$ have rigid pavement. In flexible pavement the most representative failure types alligator cracking and potholes and in the case of rigid pavement it were cracks and desintegration. The severity level of the aforementioned failures, as much for the flexible as for the rigid pavements is from mean to high, being this an indicator of the deterioration degree that the analyzed pavement sectors show. Many of the failures may be associated to pavement structural deficiencies and to inadequate conditions of the subgrade soils.
\end{abstract}

Keywords: Failure, visual inspection, pavement, severity, deterioration.

\section{INTRODUCCIÓN}

Según Hernández (2015) las vías son una variable fundamental en los procesos que impulsan el desarrollo de un país y por lo tanto contribuyen en el mejoramiento de la calidad de vida de sus habitantes, teniendo como fin permitir que las mercancías y las personas se movilicen con facilidad $y$ seguridad (Pinilla, 2007).

El estado de las vías de nuestro país es un aspecto relevante en el desarrollo del mismo (León y Hernández, 2016), el deterioro constante de las vías es generado principalmente por los agentes climáticos característicos de cada región, los daños en las redes de acueducto y alcantarillado, la falta de mantenimiento por parte de la administración pública y el tráfico de vehículos para los cuales no fueron diseñadas las vías. (Álvarez y Páez, 2014)

Como lo definen Valdés, Pérez y Calabi (2012) el pavimento es una de las partes de la carretera que requiere la mayor inversión de recursos económicos, tanto en su etapa de construcción como en su etapa de mantenimiento, siendo muy común que la segunda etapa se desarrolle en lapsos de tiempo muy grandes, llevando al deterioro progresivo de las estructuras de pavimento (Salomón, 2006).

Las condiciones en las que se desempeña un pavimento, sea este rígido o flexible, van variando con el paso de los años, siendo óptimas en edades tempranas y deficientes cuando estos han cumplido un periodo de vida útil. En la figura 1 se puede apreciar como a medida que envejece el pavimento este se va deteriorando, manifestándose este deterioro en diferentes tipos de fallos, los cuales hacen que el nivel de servicio de una vía se disminuya notablemente (Picado, 2016). 


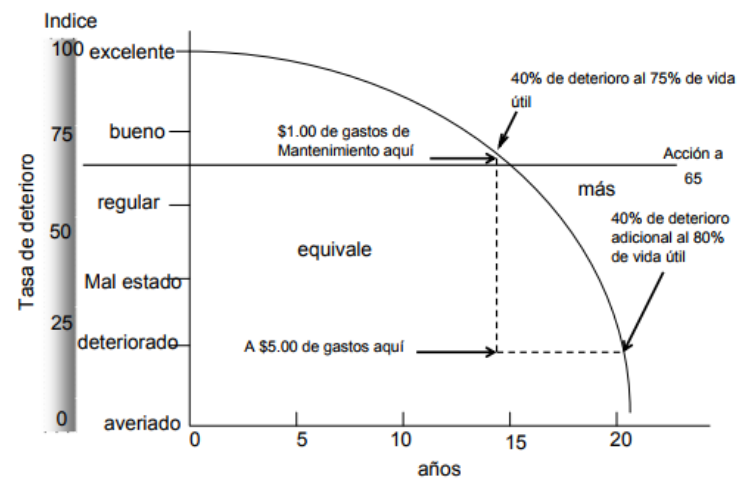

Figura 1. Curva de deterioro del pavimento

Fuente: (De Solminihac, 2001)

Por lo anterior reviste especial importancia hacer un diagnóstico del estado de la estructura del pavimento y se pueda tener criterio para definir el tipo de intervención que este requiere, además de la magnitud de los recursos necesarios para la misma (Buitrago y Cano, 2011).

Para establecer el estado superficial de un pavimento se pueden implementar varias metodologías como la PCI y la VIZIR (Pinilla, 2007); siendo esta última la adoptada por el INVIAS para el caso de pavimentos flexibles, desarrollando tanto un manual para inspección de este tipo de pavimentos como para el caso de pavimentos rígidos (Cardona y Prieto, 2014).

Una de las metodologías que se ha aplicado en la investigación realizada se apoyó en la inspección visual, la cual como lo definen (Echaveguren et al., 2001) es una metodología no invasiva que permite realizar la evaluación de estado de un pavimento, permitiendo identificar $y$ caracterizar los tipos de fallos presentes en un pavimento y su nivel de severidad.
Con esta metodología se abordó el análisis de varios de los principales tramos viales de la ciudad de Ocaña, para establecer los fallos típicos presentes en los pavimentos tanto flexibles como rígidos, que componen dichos tramos. Determinando además el nivel de severidad en que cada fallo se presenta.

\section{METODOLOGÍA}

El proyecto de investigación es de tipo descriptivo, la población estudiada son las vías del perímetro urbano del municipio de Ocaña, Norte de Santander y la muestra integra las principales vías de la malla vial de este.

El proyecto se desarrolló en cuatro fases:

Fase 1. Consistió en la inspección visual para el reconocimiento de las principales rutas por donde transita el transporte público y particular del municipio de Ocaña.

Fase 2. En esta se realizó el diseño de los formatos para diligenciamiento en campo de la información recolectada, tanto para pavimentos rígidos y flexibles; dichos formatos están basados en los manuales de inspección del INVIAS. En la Figura 2 se aprecia el formato para el caso de pavimento rígido.

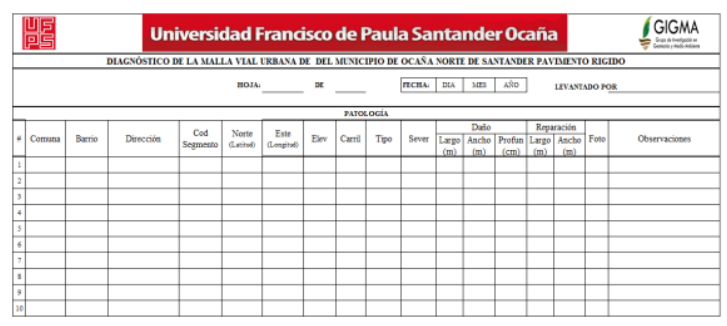

Figura 2. Formato de Pavimento Rígido Fuente: GIGMA, 2017 (adapto de INVIAS) 
Fase 3. Se realizó la Planeación y ejecución de las visitas de campo necesarias para la recolección de información, que en un principio consistió en enumerar y dar un código de referencia a las vías $\mathrm{y}$, el diligenciamiento del formato para evaluación de los fallos en las estructuras de pavimento.

Fase 4. En esta etapa se analizaron y tabularon los datos obtenidos en el trabajo de campo. El proyecto incluirá una quinta fase, que consiste en la elaboración de un sistema de información geográfica que permita hacer consulta para la planeación y priorización de los programas de mantenimiento por parte del municipio.

\section{RESULTADOS}

Una vez realizada la evaluación de las condiciones de los pavimentos, mediante inspección visual, se registraron $9.277 \mathrm{ml}$ conformados por $6.226 \mathrm{ml}$ de pavimento rígido y $3.051 \mathrm{ml}$ de pavimento flexible. En total se realizó el inventario de 3 rutas y 14 segmentos, en donde la ruta se consideró un tramo vial por donde se moviliza un número importante de vehículos de transporte público y particular y se estableció como segmento a un tramo de vía por el cual el flujo del tráfico es menor.

Una vez analizada toda la información se determinó lo siguiente:

3.1. Tipos de fallos en los pavimentos flexibles.

En la figura 3 se aprecia el porcentaje en que se presentan los tipos de fallos en estos pavimentos. El tipo de fallo piel de cocodrilo se presenta en un $10.32 \%$, seguido de los baches con un $5.06 \%$, separación de la berma con un $0.63 \%$, descaramiento con un $0.39 \%$, fisuras (longitudinales y transversales) con un $0.16 \%$ y los hundimientos con un $0.08 \%$. En la Figura 4 se aprecia el tipo de fallo piel de cocodrilo.

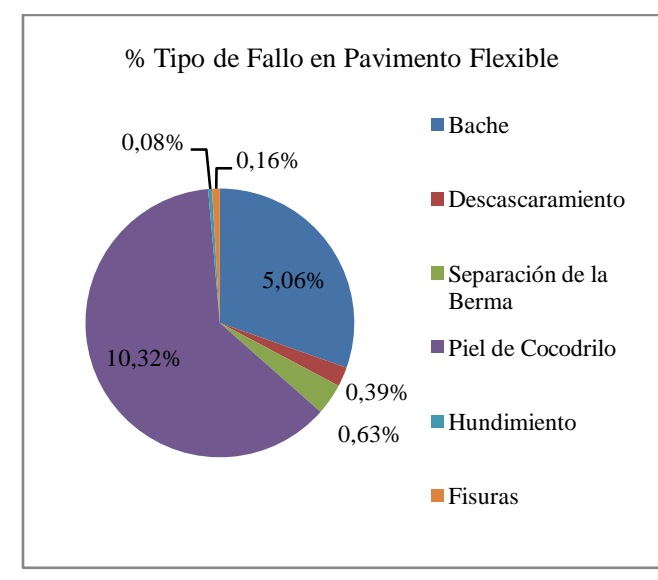

Figura 3. \%Tipo de Fallo en pavimento flexible.

Fuente: GIGMA, 2017

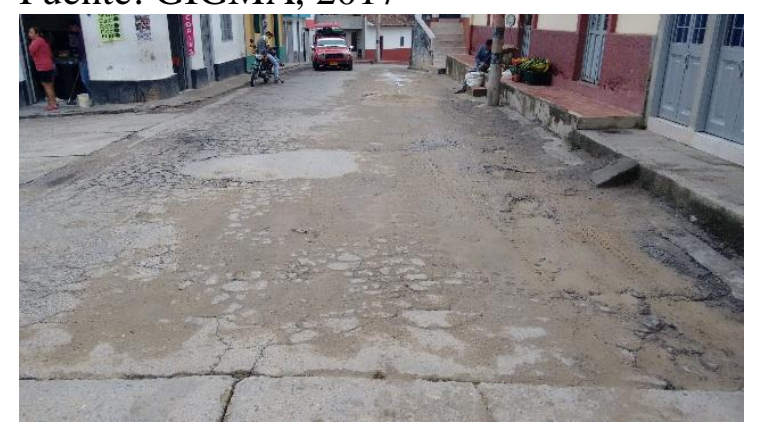

Figura 4. Fallo típico en pavimento flexible. Fuente: GIGMA, 2017

En la figura 5 se aprecia el tipo de fallo según su severidad: alta, media y baja. El tipo de fallo piel de cocodrilo representa un área afectada de $970.44 \mathrm{~m}^{2}$ de los cuales el $74,5 \%$ es de severidad alta, lo cual indica que son fisuras que han evolucionado con una abertura mayor $3 \mathrm{~mm}$ (INVIAS, 2006), seguido de $13,6 \%$ de severidad media y $11,8 \%$ de severidad baja.

Otro tipo de fallo es la pérdida de la capa de la estructura, representada en los baches y descascaramiento. En los sectores con baches un $82,3 \%$ presentan severidad alta, que son baches con profundidades mayor a $50 \mathrm{~mm}$ (INVIAS, 2006), seguido de 17,53\% de severidad media y $0,2 \%$ de severidad baja. Para el tipo de fallo descascaramiento el $100 \%$ es de severidad media. Además se 
registra casos de deformaciones (hundimiento), todos con un $100 \%$ severidad baja. Para el caso de las fisuras el $100 \%$ son de severidad media y la separación de la berma (clasificada como otro tipo de daño) con un $100 \%$ de severidad media.

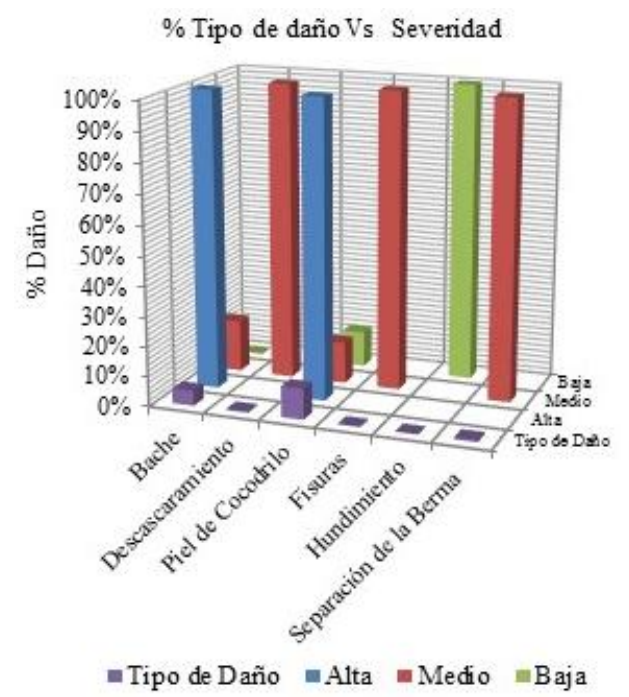

Figura 5. \% Tipo de daño según su nivel de severidad.

\section{Fuente: GIGMA, 2017}

3.2. Tipos de fallos en pavimento rígido. En la figura 6 se aprecia el porcentaje en que se presentan los tipos de fallos en los tramos viales con este tipo de pavimento. El tipo de fallo grieta (grietas en las esquinas, grietas transversales y grietas longitudinales) se presenta con un $25,8 \%$, desintegración con un 20.1\%, parches con un $17,2 \%$, descascaramiento con un $5,9 \%$, deterioro del sello con un $5,1 \%$, fisuras por retracción con un $2,4 \%$, bache con un $2,3 \%$, desportillamiento de juntas con un $2,2 \%$, escalonamiento de juntas con un $0,2 \%$ y pulimiento con un $0,2 \%$. En la figura 7 se muestra el fallo tipo grieta con nivel de severidad alto.

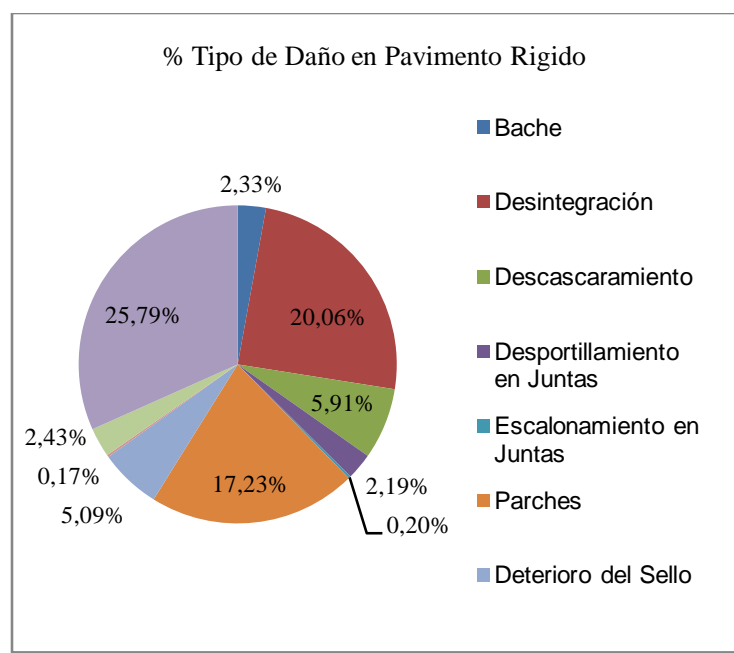

Figura 6. \%Tipo de Daño en pavimento rígido.

Fuente: GIGMA, 2017

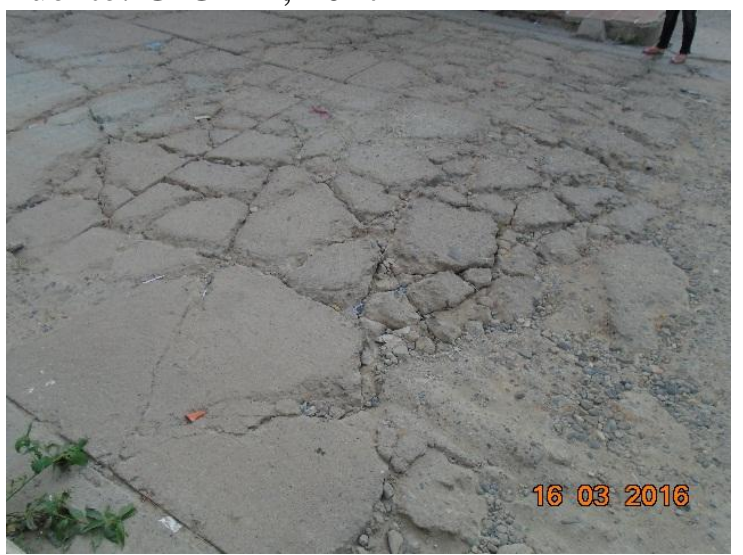

Figura 7. Fallo típico en pavimento rígido.

Fuente: GIGMA, 2017

En la figura 8 se aprecia el tipo de fallo según su severidad: alta, media y baja. Para el caso de las las grietas el $51,6 \%$ son severidad alta, el 26,3\% de severidad media y $22,1 \%$ de severidad baja. Los baches representan un área de 2356,12 $\mathrm{m}^{2}$ de los cuales $98,6 \%$ son de severidad alta, $1,2 \%$ de severidad baja y $0,2 \%$ de severidad baja.

Otro tipo de fallo es la desintegración que representa un área afectada de $3.556 \mathrm{~m}^{2}$, con 95,2\% de severidad media, $4 \%$ de severidad baja y $0,08 \%$ de severidad alta; los parches representa un área afectada con $469 \mathrm{~m}^{2}$, con un $91,8 \%$ de severidad bajo, seguido de $5 \%$ 
de severidad medio y $3,2 \%$ de severidad alta. Los descascaramientos representan un área afectada de $2.405 \mathrm{~m}^{2}$, con un $93,9 \%$ de severidad baja, 3,7\% de severidad media y 2,5\% de severidad alta.; deterioro del sello representan un área afectada con $317,11 \mathrm{~m}^{2}$, con un $58,1 \%$ de severidad alta, $32,1 \%$ de severidad media y $9,9 \%$ de severidad baja.

En el caso de las fisuras por retracción representan un área afectada $120,55 \mathrm{~m}^{2}$, con un $64,7 \%$ de severidad baja, $30,7 \%$ de severidad media y $4,49 \%$ de severidad alta; desportillamiento de juntas representa un $136,54 \mathrm{ml}$ afectados, con un $100 \%$ de severidad media; el escalonamiento de juntas presenta un $100 \%$ de severidad media; pulimiento con un $85,2 \%$ de severidad media y $14,8 \%$ de severidad alta.

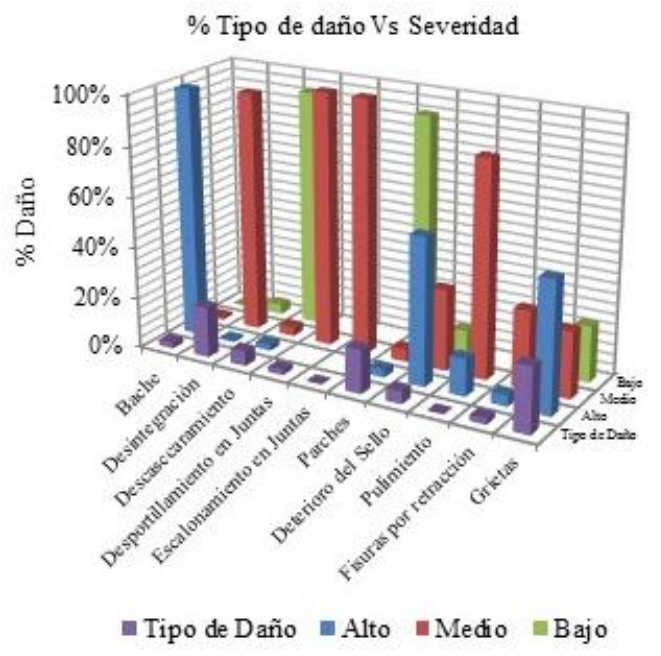

Figura 8. \%Tipo de daño según su nivel de severidad.

Fuente: GIGMA, 2017

\section{CONCLUSIONES.}

Se realizó inventario a $9.277 \mathrm{ml}$ de las principales vías del municipio de Ocaña, encontrándose que $3051 \mathrm{ml}$ (32.88\%) corresponden a pavimentos flexibles y 6226 $\mathrm{ml}(67.11 \%)$ a pavimentos rígidos.

De los tramos viales en pavimento flexible un $16.62 \%$ presentan deterioro, representado en algún tipo de fallo. Para el caso de pavimento rígido el $81.39 \%$ de los tramos presenta algún grado de deterioro. Indicando lo anterior que los pavimentos flexibles requieren menos grado de intervención.

En los pavimentos flexibles analizados, los tipos de fallos encontrados fueron: baches, descascaramiento, separación de la berma, piel de cocodrilo, hundimiento y fisura.

De los fallos más representativos encontrados en los tramos de pavimento flexible se tiene que un $10,32 \%$ pertenecen al tipo de fallo piel de cocodrilo y que un $5,06 \%$ a baches. Las principales causas de formación de piel de cocodrilo pueden ser el inadecuado diseño de la estructura, deformación en la sub-rasante, incorrecto diseño de mezcla, deficiencia en los procesos constructivos; para el caso de los baches puede ser causados por espesor de las capas de la estructura pavimento inferior al requerido o por presencia de materiales en la sub-rasante con alta plasticidad $y$ deformabilidad.

En los pavimentos rígidos analizados, los tipos fallos encontrados fueron: baches, desintegración, descascaramiento, desportillamiento de juntas, escalonamiento de juntas, parches, deterioro del sello, pulimiento, fisuras por retracción y grietas. 
De los fallos más representativos encontrados en los tramos de pavimento rígido se tiene que un $25,8 \%$ pertenecen al tipo de fallo grietas y un $20,1 \%$ a la desintegración. Las principales causas de formación de grietas pueden ser: deformaciones verticales de las capas apoyo y sub-brasante, exceso de tráfico e inadecuado diseño de la estructura; para la desintegración esta puede ser causada por resistencia baja de los concretos, incorrecto diseño de mezcla y/o procesos constructivos.

\section{FINANCIACIÓN}

Los recursos para el desarrollo de este proyecto de investigación fueron dados por División de Investigación y Extensión de la Universidad Francisco de Paula Santander Ocaña, como parte de la financiación del proyecto titulado Implementación de un sistema de información geográfico para el diagnóstico de la malla vial urbana del municipio de Ocaña N.S.

\section{AGRADECIMIENTO}

Se extiende cordial agradecimientos a los estudiantes de las asignaturas de práctica profesional del programa de ingeniería civil de la Universidad Francisco de Paula Santander, a la ingeniera Sandra Duran Duran y al ingeniero Agustín Macgregor por su colaboración.

\section{BIBLIOGRAFÍA}

Álvarez, E. y Páez, J. (2014). Diagnóstico de la red vial de los barrios Santa Clara, Dos de Octubre y Villa Paraíso del municipio de Ocaña Norte de Santander.
(Tesis de pregrado). Recuperado de repositorio.ufpso.edu.co:8080/dspaceufpso/h andle/123456789/372

Buitrago, J. y Cano, J. (2011). Análisis comparativo de metodologías de auscultación de pavimentos flexibles. (Tesis de especialización). Recuperado de http://repository.unimilitar.edu.co/bitstream/ 10654/3655/2/BuitragoVelandiaJohanaAstri d2011.pdf

Cardona, C. y Prieto S. (2014). Comparación metodologías INVIAS y metodología Mexicana (Instituto Mexicano SEDESOL) para evaluación de condición superficial de estructuras de pavimento (Tesis de especialización). Recuperado de http://repository.unimilitar.edu.co/bitstream/ 10654/12862/1/DOCUMENTO\%20DE\%20 GRADO.pdf

De Solminihac, H. (2001). Gestión de Infraestructura Vial. $2^{a}$ edición. Santiago, Chile: Ediciones Universidad Católica de Chile.

Echaveguren, T., Vargas, S., Concha, E. y Soto, A. (2001). Metodología de inspección visual para sistema de gestión de pavimentos urbanos SIGMAP. Recuperado de http://www2.udec.cl/ provial/trabajos_pdf/4 6Echavegurengestionpavimentosurbanos.pdf

Hernández-Atencia, Y. (2015). Caracterización patológica de los pavimentos en las rutas de buses y vías principales de Ibagué. (Documento de docencia No. 4). Bogotá: Ediciones Universidad Cooperativa de Colombia. doi: http://dx.doi.org/10.16925/greylit.1145.

INVIAS. (2006). Estudio e investigación del estado actual de las obras de la red nacional de carreteras. Manual para la inspección visual de pavimentos flexibles. Bogotá. 
León, E. y Hernández, A. (2015). Estudio de fallas presentadas en el pavimento rígido de la calle humareda del municipio de Rio de Oro, Cesar. (Tesis de pregrado). Recuperado de http://repositorio.ufpso.edu.co:8080/dspaceu fpso/bitstream/123456789/1008/1/28565.pdf

Muñoz, G. P. (2016). Desarrollo de curvas de deterioro para pavimento flexible y factor de incertidumbre. Infraestructura Vial, 18(31). Recuperado de https://dialnet.unirioja.es/servlet/articulo?co digo $=5793106$

Pinilla, J. (2007). Auscultación, calificación del estado superficial y evaluación económica de la carretera sector puente de La Libertad - Malteria desde K0+OOO hasta el K6+000 (código 5006) (Tesis de pregrado). Recuperado de http://www.bdigital.unal.edu.co/1982/1/julia nandrespinillavalencia.2007.pdf

Salomón, D. (2006). Conservación de pavimentos: conservando la inversión del patrimonio vial. Asfáltica, Número 4, Asociación Mexicana del Asfalto. Recuperado de http://www.pavementpreservationsystems.co $\mathrm{m} /$ publications/Conservacion-dePavimentos,Conservando-La-Inversion-delPatrimonio-Vial.pdf

Valdés, G., Pérez, F., y Calabi, A. (2012). La fisuración en pavimentos asfálticos y sus mecanismos de deterioro. Revista Ingeniería de Obras Civiles, (1), 27-37. Recuperado de http://rioc.cl/index.php/RIOC/article/view/3 